TRANSACTIONS OF THE

AMERICAN MATHEMATICAL SOCIETY

Volume 356, Number 9, Pages 3545-3556

S 0002-9947(03)03405-6

Article electronically published on December 15, 2003

\title{
NONLINEARIZABLE ACTIONS OF DIHEDRAL GROUPS ON AFFINE SPACE
}

\author{
KAYO MASUDA
}

\begin{abstract}
Let $G$ be a reductive, non-abelian, algebraic group defined over $\mathbb{C}$. We investigate algebraic $G$-actions on the total spaces of non-trivial algebraic $G$-vector bundles over $G$-modules with great interest in the case that $G$ is a dihedral group. We construct a map classifying such actions of a dihedral group in some cases and describe the spaces of those non-linearizable actions in some examples.
\end{abstract}

\section{INTRODUCTION}

Let $G$ be a reductive complex algebraic group. When $G$ is non-abelian, it is well-known that there exist non-linearizable actions of $G$ on complex affine space $\mathbb{A}^{n}$ for $n \geq 4$, i.e., algebraic actions of $G$ on $\mathbb{A}^{n}$ which are not conjugate to linear actions under polynomial automorphisms of $\mathbb{A}^{n}$. It is remarkable that non-linearizable actions on $\mathbb{A}^{n}$ known so far are all obtained from non-trivial algebraic $G$-vector bundles over $G$-modules. An algebraic $G$-vector bundle over a $G$-variety $X$ is defined to be an algebraic vector bundle $p: E \rightarrow X$, where $E$ is a $G$-variety, the projection $p$ is $G$-equivariant, and the morphism induced by $g \in G$ from $p^{-1}(x)$ to $p^{-1}(g x)$ is linear for all $g$ and $x \in X$. An algebraic $G$-vector bundle is called trivial if it is isomorphic to a product bundle $X \times Q \rightarrow X$ for some $G$-module $Q$. A total space of an algebraic $G$-vector bundle over a $G$-module is an affine space by the affirmative solution to the Serre conjecture by Quillen [19] and Suslin [21]. Thus, the $G$-action on a total space $E$ of a non-trivial $G$-vector bundle over a $G$-module is a candidate for a non-linearizable action on affine space. There are a couple of known conditions for such an action to be non-linearizable (Bass and Haboush 11, M. Masuda and Petrie [15]). Schwarz [20] (Kraft and Schwarz 7] for details) first showed that an algebraic $G$-vector bundle over a $G$-module $P$ can be non-trivial when the algebraic quotient of $P$ is of one dimension, and that there exist families of non-linearizable actions on affine space, by using the above conditions. After Schwarz, lots of examples of non-trivial algebraic $G$-vector bundles have been presented, and it turns out that many of the $G$-actions on their total spaces are non-linearizable (Knop 5], M. Masuda, Moser-Jauslin and Petrie [11, M. Masuda and Petrie [16]). For abelian groups, there are no known examples of non-linearizable actions on complex affine space. In fact, for an abelian group $G$, every algebraic $G$-vector bundle over

Received by the editors April 3, 2003.

2000 Mathematics Subject Classification. Primary 14R20; Secondary 14L30, 14D20.

Key words and phrases. Algebraic group action, linearization problem.

Supported by Grant-in-Aid for Young Scientists, The Ministry of Education, Culture, Sports, Science and Technology, Japan. 
a $G$-module becomes trivial by the result of M. Masuda, Moser-Jauslin and Petrie 12], so, we cannot obtain non-linearizable actions from $G$-vector bundles. There are some affirmative results for the linearizability for torus actions (e.g. BialynickiBirula [2], Kaliman, Koras, Makar-Limanov and Russell [4]); however, it remains open whether or not every algebraic action of an abelian group on $\mathbb{A}^{n}(n \geq 4)$ is linearizable. Especially for a finite abelian group $G$, e.g. for a cyclic group $\mathbb{Z} / n \mathbb{Z}$, we never know even whether any $G$-action on $\mathbb{A}^{3}$ is linearizable or not.

For finite groups, M. Masuda and Petrie [16] showed that there exists a family of non-linearizable actions of a dihedral group $D_{n}=\mathbb{Z} / n \mathbb{Z} \rtimes \mathbb{Z} / 2 \mathbb{Z}$ for $n$ even and $\geq 18$ on $\mathbb{A}^{4}$. They considered $D_{n}$-actions derived from algebraic $D_{n}$-vector bundles which become trivial by adding certain trivial bundles, and showed that those actions form a family in some cases. Later, Mederer [18] showed that nontrivial algebraic $D_{n}$-vector bundles form a huge family of infinite dimension for $n$ odd and $\geq 3$. In this article, we investigate $G$-actions derived from non-trivial algebraic $G$-vector bundles. We are most interested in the case that $G$ is a dihedral group. We present a new condition for such $D_{n}$-actions to be non-linearizable and construct a map which classifies such non-linearizable $D_{n}$-actions without imposing triviality on $D_{n}$-vector bundles under the addition of certain trivial bundles. We also describe the spaces of those non-linearizable $D_{n}$-actions in some examples.

\section{FAMILIES OF NON-LINEARIZABLE ACTIONS}

Let $G$ be a reductive, non-abelian algebraic group and let $Z$ be an affine $G$ variety. We denote by $\mathbb{C}[Z]$ the coordinate ring of $Z$ and by $\mathbb{C}[Z]^{G}$ the ring of invariants. The algebraic quotient $Z / / G$ is the affine variety defined by $Z / / G=$ Spec $\mathbb{C}[Z]^{G}$ and the quotient morphism $\pi_{Z}: Z \rightarrow Z / / G$ is the morphism corresponding to the inclusion $\mathbb{C}[Z]^{G} \hookrightarrow \mathbb{C}[Z]$. Let $P$ and $Q$ be $G$-modules and let $X \subseteq P$ be a $G$-subvariety containing the origin of $P$. We denote by $\operatorname{Vec}_{G}(X, Q)$ the set of algebraic $G$-vector bundles over $X$ whose fiber over the origin is isomorphic to $Q$, and $\operatorname{by~} \operatorname{Vec}_{G}(X, Q)$ the set of $G$-isomorphism classes in $\operatorname{Vec}_{G}(X, Q)$. An element $E \rightarrow X$ of $\operatorname{Vec}_{G}(X, Q)$ is represented by the total space $E$, and the isomorphism class of $E \in \operatorname{Vec}_{G}(X, Q)$ is denoted by $[E]$. The $\operatorname{set} \operatorname{Vec}_{G}(X, Q)$ is called trivial if $\operatorname{Vec}_{G}(X, Q)$ consists of the unique class $\left[\Theta_{Q}\right]$, where $\Theta_{Q}$ denotes the product bundle with fiber $Q$. When $\operatorname{dim} P / / G=1$, Schwarz [20] showed that $\operatorname{Vec}_{G}(P, Q)$ has an additive group structure and is isomorphic to a vector group $\mathbb{C}^{q}$ for a nonnegative integer $q$. Mederer [18] (cf. [8]) extended the result of Schwarz to the case where the base space is a $G$-equivariant affine cone $X$ with $\operatorname{dim} X / / G=1$. When $\operatorname{dim} P / / G \geq 2, \operatorname{Vec}_{G}(P, Q)$ can be non-trivial and of countably or uncountably infinite dimension $([9,[10,18])$.

We assume that $\operatorname{Vec}_{G}(P, Q)$ is non-trivial. Let $E \in \operatorname{Vec}_{G}(P, Q)$. The following are the known conditions for the $G$-action on the total space $E$ to be nonlinearizable.

Proposition 2.1. Let $E, E^{\prime} \in \operatorname{Vec}_{G}(P, Q)$.

(1) ([15]) Suppose that there exists a subgroup $H$ of $G$ such that $(P \oplus Q)^{H}=P$. Then $E$ and $E^{\prime}$ are isomorphic as $G$-varieties if and only if $E$ and the pullback $\varphi^{*} E^{\prime}$ are isomorphic as $G$-vector bundles for some $G$-automorphism $\varphi$ of $P$.

(2) ([1]) If the Whitney sum $E \oplus \Theta_{P}$ is non-trivial, then the $G$-action on $E$ is non-linearizable. 
Let $\operatorname{VAR}_{G}(P, Q)$ be the set of $G$-isomorphism classes of affine $G$-spaces represented as the total spaces of elements of $\operatorname{Vec}_{G}(P, Q)$. The group $\operatorname{Aut}(P)^{G}$ of $G$-equivariant automorphisms of $P$ acts on $\operatorname{Vec}_{G}(P, Q)$ by pull-backs. There exists a surjection $\Psi$ from the orbit space of $\operatorname{Vec}_{G}(P, Q)$ under the action of $\operatorname{Aut}(P)^{G}$ to $\operatorname{VAR}_{G}(P, Q)$. Under the assumption in Proposition $2.1(1), \Psi$ is an isomorphism.

Example 2.1. Let $G=O(2)=\mathbb{C}^{*} \rtimes \mathbb{Z} / 2 \mathbb{Z}$ and let $V_{m}(m \geq 1)$ be a twodimensional $O(2)$-module such that

$$
\begin{aligned}
& \lambda(x, y)=\left(\lambda^{m} x, \lambda^{-m} y\right) \quad \text { for } \lambda \in \mathbb{C}^{*}, \\
& \tau(x, y)=(y, x) \quad \text { for the generator } \tau \in \mathbb{Z} / 2 \mathbb{Z} .
\end{aligned}
$$

Then $V_{m} / / O(2)=\operatorname{Spec} \mathbb{C}[t]=\mathbb{A}^{1}$, where $t=x y$, and $\operatorname{Aut}\left(V_{m}\right)^{G}=\mathbb{C}^{*}$, namely, $\operatorname{Aut}\left(V_{m}\right)^{G}$ consisits of scalar multiplications.

Let $n$ be odd. Then $\operatorname{Vec}_{G}\left(V_{2}, V_{n}\right) \cong \mathbb{C}^{(n-1) / 2}$ and the Whitney sum with $\Theta_{V_{2}}$ induces an isomorphism between $\operatorname{Vec}_{G}\left(V_{2}, V_{n}\right)$ and $\operatorname{Vec}_{G}\left(V_{2}, V_{n} \oplus V_{2}\right)([20])$. By Proposition 2.1 (1) or (2), if $E \in \operatorname{Vec}_{O(2)}\left(V_{2}, V_{n}\right)$ is non-trivial, then the $O(2)$-action on $E$ is non-linearizable. We shall describe $\operatorname{VAR}_{O(2)}\left(V_{2}, V_{n}\right)$. Since $\left(V_{2} \oplus V_{n}\right)^{\mathbb{Z} / 2 \mathbb{Z}}=$ $V_{2}$, where $\mathbb{Z} / 2 \mathbb{Z}$ is a subgroup of $\mathbb{C}^{*} \subset O(2)$, it follows from Proposition 2.1 (1) that

$$
\operatorname{VAR}_{O(2)}\left(V_{2}, V_{n}\right) \cong \operatorname{Vec}_{O(2)}\left(V_{2}, V_{n}\right) / \mathbb{C}^{*}
$$

In order to look at the action of $\operatorname{Aut}\left(V_{2}\right)^{G}=\mathbb{C}^{*}$ on $\operatorname{Vec}_{G}\left(V_{2}, V_{n}\right)$, recall the isomorphism $\operatorname{Vec}_{G}\left(V_{2}, V_{n}\right) \cong \mathbb{C}^{(n-1) / 2}$. For the details, we refer to Kraft and Schwarz [7]. Let $F=\pi_{V_{2}}^{-1}(1)$, which is the $G$-subvariety of $V_{2}$ defined by $x y=1$. Then $F \cong G / H$, where $H=\mathbb{Z} / 2 \mathbb{Z} \rtimes \mathbb{Z} / 2 \mathbb{Z}$, and $V_{n}$ is multiplicity-free with respect to $H$, namely, each irreducible $H$-module occurs in $V_{n}$ with multiplicity at most one when $V_{n}$ is viewed as an $H$-module. We set $\mathfrak{m}=\operatorname{Mor}\left(F, \text { End } V_{n}\right)^{G}$, the module of $G$-equivariant morphisms from $F$ to End $V_{n}$. Let $\mathbb{B}=\operatorname{Spec} \mathbb{C}[s]$ be the double cover of $\mathbb{A}^{1}=\operatorname{Spec} \mathbb{C}[t]$, where $s^{2}=t$. Then the group $\Gamma:=\{ \pm 1\}$ acts on $\mathbb{B}$ and on $F$ by scalar multipication. We denote by $\mathbb{B} \times_{\Gamma} F$ the quotient of $\mathbb{B} \times F$ by $\Gamma$ which acts by $(b, f) \mapsto\left(b \gamma, \gamma^{-1} f\right)$ for $\gamma \in \Gamma, b \in \mathbb{B}$, and $f \in F$. The group $G$ acts on $\mathbb{B} \times_{\Gamma} F$ through $F$. We define a $G$-equivariant morphism $\varphi$ by

$$
\begin{aligned}
\varphi: \mathbb{B} \times_{\Gamma} F & \rightarrow V_{2}, \\
{[b, f] } & \mapsto b f,
\end{aligned}
$$

which is a $G$-isomorphism from $(\mathbb{B}-\{0\}) \times_{\Gamma} F$ onto $V_{2}-\pi_{V_{2}}^{-1}(0)$. Note that $\mathbb{C}\left[\mathbb{B} \times{ }_{\Gamma} F\right]^{G} \cong \mathbb{C}[\mathbb{B}]^{\Gamma}=\mathbb{C}[t]=\mathbb{C}\left[V_{2}\right]^{G}$. The morphism $\varphi$ induces a homomorphism

$$
\begin{aligned}
\varphi_{\#}: \operatorname{Mor}\left(V_{2}, \text { End } V_{n}\right)^{G} & \rightarrow \operatorname{Mor}\left(\mathbb{B} \times_{\Gamma} F, \text { End } V_{n}\right)^{G} \\
& =\operatorname{Mor}(\mathbb{B}, \mathfrak{m})^{\Gamma}=: \mathfrak{m}(\mathbb{B})^{\Gamma} .
\end{aligned}
$$

The modules $\operatorname{Mor}\left(V_{2} \text {, End } V_{n}\right)^{G}$ and $\mathfrak{m}(\mathbb{B})^{\Gamma}$ are finite free modules over $\mathbb{C}[t]$. In fact, a basis of $\operatorname{Mor}\left(V_{2} \text {, End } V_{n}\right)^{G} \cong\left(\mathbb{C}\left[V_{2}\right] \otimes \text { End } V_{n}\right)^{G}$ over $\mathbb{C}[t]$ is written in a matrix form as

$$
\left\{A_{0}=\left(\begin{array}{ll}
1 & 0 \\
0 & 1
\end{array}\right), A_{1}=\left(\begin{array}{cc}
0 & x^{n} \\
y^{n} & 0
\end{array}\right)\right\}
$$

and a basis of $\mathfrak{m}(\mathbb{B})^{\Gamma} \cong(\mathbb{C}[s] \otimes \mathfrak{m})^{\Gamma}$ over $\mathbb{C}[t]$ is

$$
\left\{C_{0}=\left(\begin{array}{ll}
1 & 0 \\
0 & 1
\end{array}\right), C_{1}=s\left(\left.A_{1}\right|_{F}\right)\right\} \text {. }
$$


The module $\operatorname{Mor}\left(V_{2} \text {, End } V_{n}\right)^{G}$ (resp. $\left.\mathfrak{m}(\mathbb{B})^{\Gamma}\right)$ inherits a grading from $\mathbb{C}\left[V_{2}\right]$ (resp. $\mathbb{C}[s])$, and $\varphi_{\#}$ is a homomorphism of degree 0 . Let $\operatorname{Mor}\left(V_{2} \text {, End } V_{n}\right)_{1}^{G}\left(\operatorname{resp} \cdot \mathfrak{m}(\mathbb{B})_{1}^{\Gamma}\right)$ be the submodule of $\operatorname{Mor}\left(V_{2}, \text { End } V_{n}\right)^{G}$ (resp. $\left.\mathfrak{m}(\mathbb{B})^{\Gamma}\right)$ consisting of elements with positive degrees. Then $\operatorname{Vec}_{G}\left(V_{2}, V_{n}\right)$ is isomorphic to the quotient module $\mathfrak{m}(\mathbb{B})_{1}^{\Gamma} / \varphi_{\#} \operatorname{Mor}\left(V_{2}, \text { End } V_{n}\right)_{1}^{G}$. Since

$$
\varphi_{\#}\left(A_{0}\right)=C_{0} \quad \text { and } \quad \varphi_{\#}\left(A_{1}\right)=t^{\frac{n-1}{2}} C_{1},
$$

$\left\{t^{i-1} C_{1} ; 1 \leq i \leq \frac{n-1}{2}\right\}$ forms a $\mathbb{C}$-basis of $\mathfrak{m}(\mathbb{B})_{1}^{\Gamma} / \varphi_{\#} \operatorname{Mor}\left(V_{2} \text {, End } V_{n}\right)_{1}^{G}$, and hence

$$
\operatorname{Vec}_{G}\left(V_{2}, V_{n}\right) \cong \mathfrak{m}(\mathbb{B})_{1}^{\Gamma} / \varphi_{\#} \operatorname{Mor}\left(V_{2}, \text { End } V_{n}\right)_{1}^{G} \cong \mathbb{C}^{\frac{n-1}{2}} .
$$

Note that $\operatorname{deg}\left(t^{i-1} C_{1}\right)=2 i-1$. The scalar multiplication on $V_{2}$ corresponds to a scalar multiplication on $\mathbb{B}$ via $\varphi$. Hence $\operatorname{Vec}_{G}\left(V_{2}, V_{n}\right) \cong \bigoplus_{i=1}^{(n-1) / 2} W(2 i-1)$ as a module of $\operatorname{Aut}\left(V_{2}\right)^{G}=\mathbb{C}^{*}$, where $W(i)$ denotes the representation space of $\mathbb{C}^{*}$ with weight $i$. Thus we obtain by Proposition 2.1 (1) that

$$
\begin{aligned}
\operatorname{VAR}_{O(2)}\left(V_{2}, V_{n}\right) & \cong\left(\bigoplus_{i=1}^{(n-1) / 2} W(2 i-1)\right) / \mathbb{C}^{*} \\
& =: \quad \mathbb{P}_{*}\left(2 i-1 ; 1 \leq i \leq \frac{n-1}{2}\right) .
\end{aligned}
$$

Here $\mathbb{P}_{*}(2 i-1 ; 1 \leq i \leq(n-1) / 2)$ consisits of the "vertex" $*$ and the weighted projective space $\mathbb{P}(2 i-1 ; 1 \leq i \leq(n-1) / 2)$ of dimension $(n-3) / 2$ with weight $2 i-1$ for $1 \leq i \leq(n-1) / 2$. The "vertex" corresponds to the linearizable action and the weighted projective space to non-linearizable actions (cf. [16]).

Example 2.2. Let $G=S L_{2}$ and let $R_{n}$ be the $S L_{2}$-module of binary forms of degree $n \geq 1$. Then $\operatorname{Vec}_{G}\left(R_{2}, R_{n}\right) \cong \mathbb{C}^{\left[(n-1)^{2} / 4\right]}$ and $\operatorname{Aut}\left(R_{2}\right)^{G}=\mathbb{C}^{*}([20]$, [7] $)$. As a module of $\mathbb{C}^{*}=\operatorname{Aut}\left(R_{2}\right)^{G}, \operatorname{Vec}_{G}\left(R_{2}, R_{n}\right)$ is isomorphic to $\bigoplus_{i=1}^{n-2} m_{i} W(i)$ with multiplicity $m_{i}=\left[\frac{n-i}{2}\right]$. Suppose $n$ is odd. Then $\left(R_{2} \oplus R_{n}\right)^{\mathbb{Z} / 2 \mathbb{Z}}=R_{2}$. Hence by Proposition 2.1 (1),

$$
\begin{aligned}
\operatorname{VAR}_{S L_{2}}\left(R_{2}, R_{n}\right) & \cong\left(\bigoplus_{i=1}^{n-2} m_{i} W(i)\right) / \mathbb{C}^{*} \\
& =: \quad \mathbb{P}_{*}\left(i, m_{i} ; 1 \leq i \leq n-2\right) .
\end{aligned}
$$

In this case, the space of non-linearizable $S L_{2}$-actions is isomorphic to the weighted projective space of dimension $\left[(n-1)^{2} / 4\right]-1$ with weight $i$ of multiplicity $m_{i}$ for $1 \leq i \leq n-2$.

Example 2.3. Let $G$ be semisimple and let $\mathfrak{g}$ be the adjoint representation of $G$. Let $\Sigma$ be a system of simple roots of $G$ and $F$ an irreducible $G$-module with the highest weight $\chi$. Knop [5] constructed a map associated with $\alpha \in \Sigma$,

$$
\Phi_{\alpha}: \operatorname{Vec}_{G}(\mathfrak{g}, F) \rightarrow \operatorname{Vec}_{S L_{2}}\left(R_{2}, R_{m}\right),
$$

where $m=\langle\chi, \alpha\rangle$. The map $\Phi_{\alpha}$ is surjective if the $\alpha$-string of $\chi$ is regular ([5], [14]). We recall the construction of $\Phi_{\alpha}$. Let $T \subset G$ be a maximal torus with the Lie algebra $\mathfrak{t} \subset \mathfrak{g}$. Let $L$ be the subgroup of $G$ generated by $T$ and the root subgroups $U_{\alpha}$ and $U_{-\alpha}$. We denote by $L^{\prime}$ the commutator subgroup of $L$ and by $Z$ the center of $L$. Then $L=L^{\prime} Z$, and $L^{\prime}$ is isomorphic to $S L_{2}$ or $S O_{3}$. Let $\mathfrak{l}$ be the Lie algebra of $L$. Then $\mathfrak{l}$ is isomorphic to $\mathfrak{s l}_{2} \oplus \mathbb{C}^{n-1}$ as an $L^{\prime}$-module, 
where $n=\operatorname{rank} \mathfrak{t}$. For $E \in \operatorname{Vec}_{G}(\mathfrak{g}, F)$, the restricted bundle $\left.E\right|_{\mathfrak{r}}$ is an $L$-vector bundle with fiber $F^{\prime}$ which is $F$ viewed as an $L$-module. Take a $\xi_{0} \in \mathfrak{t}$ so that the centralizer of $\xi_{0}$ is exactly $L$, and fix it. Then $\mathfrak{a}:=\xi_{0}+\operatorname{Lie} L^{\prime} \subseteq \mathfrak{g}$ is $L$-stable and isomorphic to $\mathfrak{s l}_{2} \cong R_{2}$ as an $L^{\prime}$-variety. Since $Z$ acts trivially on $\mathfrak{l}$, hence on $\mathfrak{a},\left.E\right|_{\mathfrak{a}}$ decomposes to a Whitney sum of eigenbundles of $Z$. Let $\left(\left.E\right|_{\mathfrak{a}}\right)_{\chi}$ be the eigenbundle corresponding to the restricted weight of $\chi$ onto $Z$. Then the $L^{\prime}$-vector bundle $\left(\left.E\right|_{\mathfrak{a}}\right)_{\chi}$ is considered as an element of $\operatorname{Vec}_{S L_{2}}\left(R_{2}, R_{m}\right)$. The map $\Phi_{\alpha}$ is defined by $\Phi_{\alpha}(E)=\left(\left.E\right|_{\mathfrak{a}}\right)_{\chi}$. By the construction of $\Phi_{\alpha}, \Phi_{\alpha}$ decomposes to the maps

$$
\phi_{\alpha}: \operatorname{Vec}_{G}(\mathfrak{g}, F) \rightarrow \operatorname{Vec}_{L}\left(\mathfrak{l}, F^{\prime}\right) \rightarrow \operatorname{Vec}_{S L_{2}}\left(R_{2} \oplus \mathbb{C}^{n-1}, R_{m}\right)
$$

and

$$
\phi_{\xi_{0}}: \operatorname{Vec}_{S L_{2}}\left(R_{2} \oplus \mathbb{C}^{n-1}, R_{m}\right) \rightarrow \operatorname{Vec}_{S L_{2}}\left(R_{2}, R_{m}\right) .
$$

From the choice of $\xi_{0}, \phi_{\xi_{0}}$ is surjective. In fact, $\phi_{\xi_{0}} \circ p r^{*}=i d$, where

$$
p r^{*}: \operatorname{Vec}_{S L_{2}}\left(R_{2}, R_{m}\right) \rightarrow \operatorname{Vec}_{S L_{2}}\left(R_{2} \oplus \mathbb{C}^{n-1}, R_{m}\right)
$$

is the induced map from the projection $R_{2} \oplus \mathbb{C}^{n-1} \rightarrow R_{2}$. When $\Phi_{\alpha}$ is surjective, $\phi_{\alpha}$ is also surjective since $\phi_{\xi_{0}}$ is surjective. By [9],

$$
\operatorname{Vec}_{S L_{2}}\left(R_{2} \oplus \mathbb{C}^{n-1}, R_{m}\right) \cong \operatorname{Vec}_{S L_{2}}\left(R_{2}, R_{m}\right) \otimes_{\mathbb{C}} \mathbb{C}\left[\mathbb{C}^{n-1}\right] .
$$

Hence we obtain the following.

Theorem 2.2. Under the nontation above, if the $\alpha$-string of $\chi$ is regular, then

$$
\begin{aligned}
\phi_{\alpha}: \operatorname{Vec}_{G}(\mathfrak{g}, F) & \rightarrow \operatorname{Vec}_{S L_{2}}\left(R_{2} \oplus \mathbb{C}^{n-1}, R_{m}\right) \\
& \cong \mathbb{C}^{\left[(m-1)^{2} / 4\right]} \otimes_{\mathbb{C}} \mathbb{C}\left[y_{1}, \cdots, y_{n-1}\right]
\end{aligned}
$$

is surjective. Furthermore, if there is a subgroup $H$ such that $(\mathfrak{g} \oplus F)^{H}=\mathfrak{g}$, then $\phi_{\alpha}$ induces a surjection

$$
\operatorname{VAR}_{G}(\mathfrak{g}, F) \rightarrow\left(\mathbb{C}^{\left[(m-1)^{2} / 4\right]} \otimes_{\mathbb{C}} \mathbb{C}\left[y_{1}, \cdots, y_{n-1}\right]\right) / \mathbb{C}^{*},
$$

where $\mathbb{C}^{*}$ acts on $\mathbb{C}^{\left[(m-1)^{2} / 4\right]}$ with weight $i$ of multiplicity $m_{i}=[(m-i) / 2]$ and on $y_{i}$ with weight 1 .

Proof. The first assertion follows from the above observation. For the second assertion, note that $\operatorname{Aut}(\mathfrak{g})^{G}=\mathbb{C}^{*}([7])$. From Proposition $2.1(1)$, there is an isomorphism $\operatorname{VAR}_{G}(\mathfrak{g}, F) \cong \operatorname{Vec}_{G}(\mathfrak{g}, F) / \mathbb{C}^{*}$. Hence $\phi_{\alpha}$ induces a surjection

$$
\operatorname{VAR}_{G}(\mathfrak{g}, F) \rightarrow \operatorname{Vec}_{S L_{2}}\left(R_{2} \oplus \mathbb{C}^{n-1}, R_{m}\right) / \mathbb{C}^{*}
$$

The assertion follows from the statement in Example 2.2.

Remark. When the $\alpha$-string of $\chi$ is singular, the image of $\Phi_{\alpha}$ contains a subspace of dimension $[m / 2]([m / 2]-1) / 2([14])$.

By Theorem 2.2 and its remark, $\operatorname{Vec}_{G}(\mathfrak{g}, F)$ is of infinite dimension if $m \geq 4$ and $n \geq 2$. Furthermore, if $(\mathfrak{g} \oplus F)^{H}=\mathfrak{g}$ for a subgroup $H$, then $\operatorname{VAR}_{G}(\mathfrak{g}, F)$ is of infinite dimension.

Now, we give a new condition for the $G$-action on $E \in \operatorname{Vec}_{G}(P, Q)$ to be nonlinearizable, which is used as a basic fact in the next section.

Proposition 2.3. Let $E, E^{\prime} \in \operatorname{Vec}_{G}(P, Q)$. Suppose that there exist reductive subgroups $H$ and $K$ such that $H \subset K$ and satisfying the following conditions;

(1) $Q^{K}=Q^{H}$,

(2) $\operatorname{dim} P^{H}=1$ and $\operatorname{dim} P^{K}=0$. 
If $E \cong E^{\prime}$ as $G$-varieties, then the restricted bundles $\left.E\right|_{X}$ and $\left.\left(c^{*} E^{\prime}\right)\right|_{X}$ are isomorphic as $G$-vector bundles, where $X=\overline{G \cdot P^{H}}$ and $c$ is a scalar multiplication on $P$. In particular, if $\left.E\right|_{X}$ is a non-trivial $G$-vector bundle, then the $G$-action on $E$ is non-linearizable.

Proof. Let $\phi: E \cong E^{\prime}$ be an isomorphism of $G$-varieties. Then $\phi$ restricts to an isomorphism $\phi_{H}: E^{H} \cong E^{\prime H}$. Since $E^{H}$ and $E^{\prime H}$ are trivial $(H$-)vector bundles over $P^{H}$ with fiber $Q^{H}$ (cf. [6]), it follows that $E^{H} \cong E^{\prime H} \cong P^{H} \times Q^{H}$. Similarly, $E^{K} \cong E^{\prime K} \cong Q^{K}=Q^{H}$ since $\operatorname{dim} P^{K}=0$. Since $E^{K}$ (resp. $E^{\prime K}$ ) is a subbundle of $E^{H}$ (resp. $E^{\prime H}$ ), we get $E^{H}=E^{K} \times P^{H}$ and $E^{\prime H}=E^{\prime K} \times P^{H}$. Let $x$ be a coordinate variable of $P^{H} \times Q^{H}$ such that $P^{H}=\operatorname{Spec} \mathbb{C}[x]$. Then the ideal corresponding to $E^{K}$ is $(x)$, and the ideal for $E^{\prime K}$ is the same. Since $\phi$, hence $\phi_{H}$, maps $E^{K}$ to $E^{\prime K}$ isomorphically, the ideal $(x)$ must be fixed by the algebra isomorphism corresponding to $\phi_{H}$. This implies that $\phi_{H}$, hence $\phi$, induces an isomorphism $\bar{c}$ on $P^{H}$ such that $p_{H}^{\prime} \circ \phi_{H}=\bar{c} \circ p_{H}$, where $p_{H}: E^{H} \rightarrow P^{H}$ and $p_{H}^{\prime}: E^{\prime H} \rightarrow P^{H}$ are projections. Note that $\bar{c}$ is a scalar multiplication on $P^{H}$. Hence $\bar{c}$ extends to a scalar multiplication $c$ on $P$. Since the following diagram commutes, $\phi$ restricts to a variety isomorphism $\left.\left.E\right|_{P^{H}} \cong E^{\prime}\right|_{P^{H}}$ :

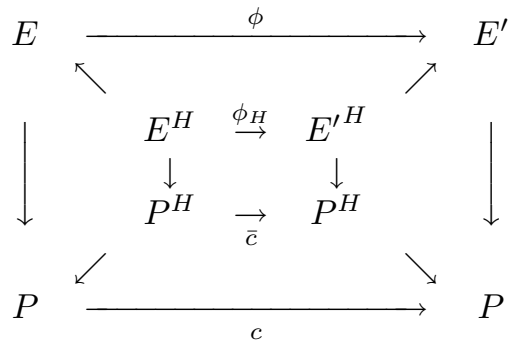

where the diagonal arrows are inclusions. Furthermore, since $\phi$ is a $G$-isomorphism, $\phi$ in fact restricts to a $G$-isomorphism $\phi_{X}:\left.\left.E\right|_{X} \rightarrow E^{\prime}\right|_{X}$ such that $p_{X}^{\prime} \circ \phi_{X}=$ $\left(\left.c\right|_{X}\right) \circ p_{X}$, where $p_{X}:\left.E\right|_{X} \rightarrow X$ and $p_{X}^{\prime}:\left.E^{\prime}\right|_{X} \rightarrow X$ are projections. Thus $\left.\left.E\right|_{X} \cong\left(c^{*} E^{\prime}\right)\right|_{X}$ as $G$-vector bundles, and the assertion follows.

Proposition 2.3 enables us to classify elements of $\operatorname{VAR}_{G}(P, Q)$.

Corollary 2.4. Under the assumption and notation in Proposition 2.3, there exists a map

$$
\Phi: \operatorname{VAR}_{G}(P, Q) \rightarrow \operatorname{Vec}_{G}(X, Q) / \mathbb{C}^{*},
$$

where the target space is the orbit space of $\operatorname{Vec}_{G}(X, Q)$ under the action of $\mathbb{C}^{*}$, which is a subgroup of $\operatorname{Aut}(X)^{G}$ consisting of scalar multiplications.

When $H$ is an isotropy group of a point $x \in P$ whose orbit is closed, then $X=\overline{G \cdot P^{H}}$ is a $G$-equivariant affine cone in $P$ with $\operatorname{dim} X / / G=1$. In this case, $\operatorname{Vec}_{G}(X, Q)$ is isomorphic to a finite-dimensional module of $\mathbb{C}^{*} \subset \operatorname{Aut}(X)^{G}$ ([18]). Hence $\operatorname{Vec}_{G}(X, Q) / \mathbb{C}^{*}$ is isomorphic to a weighted projective space with a "vertex".

Example 2.4. Let $G=O(2)$ and consider $\operatorname{Vec}_{O(2)}\left(V_{1}, V_{m}\right)$. Then applying Proposition 2.3 for $H=\mathbb{Z} / 2 \mathbb{Z}$ (the reflection subgroup) and $K=\mathbb{Z} / m \mathbb{Z} \rtimes \mathbb{Z} / 2 \mathbb{Z}$, we obtain $X=V_{1}$, and hence, a map $\operatorname{VAR}_{O(2)}\left(V_{1}, V_{m}\right) \rightarrow \operatorname{Vec}_{O(2)}\left(V_{1}, V_{m}\right) / \mathbb{C}^{*}$, which is an isomorphism. Since $\operatorname{Vec}_{O(2)}\left(V_{1}, V_{m}\right) \cong \bigoplus_{i=1}^{m-1} W(2 i)$ ([7]), we have 
(cf. [16], [17])

$$
\begin{aligned}
\operatorname{VAR}_{O(2)}\left(V_{1}, V_{m}\right) & \cong\left(\bigoplus_{i=1}^{m-1} W(2 i)\right) / \mathbb{C}^{*} \\
& =\mathbb{P}_{*}(2 i ; 1 \leq i \leq m-1) .
\end{aligned}
$$

We apply Proposition 2.3 and its corollary for dihedral groups and classify nonlinearizable actions of dihedral groups in the next section.

\section{Non-LINEARIZABLE ACTIONS OF DIHEDRAL GROUPS}

In this section, we investigate non-linearizable actions of dihedral groups. Let $G$ be a dihedral group $D_{n}=\mathbb{Z} / n \mathbb{Z} \rtimes \mathbb{Z} / 2 \mathbb{Z}$ for $n>2$. By considering $D_{n}$ as a finite subgroup of $O(2)=\mathbb{C}^{*} \rtimes \mathbb{Z} / 2 \mathbb{Z}$, an $O(2)$-module $V_{m}$ is naturally considered as a $D_{n}$-module. Since $V_{m} \cong V_{|m-n|}$ as a $D_{n}$-module, we may assume $m \leq n / 2$; otherwise $m=n$. Let $k$ be a positive integer such that $(k, n)=1$ and $k \leq n / 2$. Let $\{x, y\}$ be a coordinate system of $V_{k}$ as in Example 2.1. Then $V_{k} / / D_{n}=\operatorname{Spec} \mathbb{C}[t, u]$,

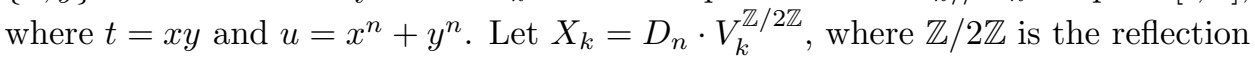
subgroup. Then $X_{k}$ is the $D_{n}$-subvariety of $V_{k}$ defined by $x^{n}-y^{n}=0$ for $n$ odd, and $x^{n / 2}-y^{n / 2}=0$ for $n$ even. The algebraic quotient of $X_{k}$ is

$$
X_{k} / / D_{n}= \begin{cases}\operatorname{Spec} \mathbb{C}[t, u] /\left(u^{2}-4 t^{n}\right) & \text { for } n \text { odd, } \\ \operatorname{Spec} \mathbb{C}[t] & \text { for } n \text { even. }\end{cases}
$$

The variety $X_{k}$ is the $D_{n}$-equivariant affine cone in $V_{k}$ with one-dimensional quotient. Hence $\operatorname{Vec}_{D_{n}}\left(X_{k}, V_{m}\right) \cong \mathbb{C}^{q}$ for some $q([18$, [8]).

We shall classify elements of $\operatorname{VAR}_{D_{n}}\left(V_{k}, V_{m}\right)$ under a certain condition.

Proposition 3.1. Let $E, E^{\prime} \in \operatorname{Vec}_{D_{n}}\left(V_{k}, V_{m}\right)$ and let $X_{k}$ be as above. Suppose that $(m, n)>1$. Then, if $E \cong E^{\prime}$ as $D_{n}$-varieties, then the restricted bundles $\left.E\right|_{X_{k}}$ and $\left.\left(c^{*} E^{\prime}\right)\right|_{X_{k}}$ are isomorphic as $D_{n}$-vector bundles, where $c$ is a scalar multiplication on $V_{k}$.

Proof. By taking $H=\mathbb{Z} / 2 \mathbb{Z}$ (the reflection subgroup) and $K=\mathbb{Z} / p \mathbb{Z} \rtimes \mathbb{Z} / 2 \mathbb{Z}$, where $p=(m, n)$ in Proposition 2.3, the assertion follows.

Under the assumption in Proposition 3.1, there exists a map

$$
\Phi_{k, m}: \operatorname{VAR}_{D_{n}}\left(V_{k}, V_{m}\right) \rightarrow \operatorname{Vec}_{D_{n}}\left(X_{k}, V_{m}\right) / \mathbb{C}^{*} .
$$

Let $i_{k}^{*}: \operatorname{Vec}_{D_{n}}\left(V_{k}, V_{m}\right) \rightarrow \operatorname{Vec}_{D_{n}}\left(X_{k}, V_{m}\right)$ be the restriction induced by the inclusion $i_{k}: X_{k} \hookrightarrow V_{k}$. There exists a sequence

$$
\operatorname{Vec}_{O(2)}\left(V_{k}, V_{m}\right) \stackrel{d_{n}}{\rightarrow} \operatorname{Vec}_{D_{n}}\left(V_{k}, V_{m}\right) \stackrel{i_{k}^{*}}{\rightarrow} \operatorname{Vec}_{D_{n}}\left(X_{k}, V_{m}\right)
$$

where $d_{n}$ is the group restriction.

Theorem 3.2 (cf. 16]). Let $n$ be odd, and let $k=2$ and $m=n$ in the notation above.

(1) The composite map $i_{2}^{*} \circ d_{n}: \operatorname{Vec}_{O(2)}\left(V_{2}, V_{n}\right) \rightarrow \operatorname{Vec}_{D_{n}}\left(X_{2}, V_{n}\right)$ is injective and

$$
\operatorname{Im}\left(i_{2}^{*} \circ d_{n}\right) \cong \mathbb{C}^{\frac{n-1}{2}}
$$

(2) The image of $\Phi_{2, n}$ is isomorphic to $\mathbb{P}_{*}(2 i-1 ; 1 \leq i \leq(n-1) / 2)$. 
(3) The map $\operatorname{VAR}_{O(2)}\left(V_{2}, V_{n}\right) \rightarrow \operatorname{VAR}_{D_{n}}\left(V_{2}, V_{n}\right)$ is injective. Hence, if $E \in$ $\operatorname{Vec}_{O(2)}\left(V_{2}, V_{n}\right)$ is a non-trivial $O(2)$-vector bundle, then the $D_{n}$-action on $E$ is non-linearizable.

Proof. (1) By applying the method of Mederer, we can show that $\operatorname{Vec}_{D_{n}}\left(X_{2}, V_{n}\right)$ is isomorphic to a vector group $\mathbb{C}^{n-1}$. For the detailed argument, we refer to Mederer [18. We shall give a basis of $\operatorname{Vec}_{D_{n}}\left(X_{2}, V_{n}\right) \cong \mathbb{C}^{n-1}$. We use the notation in Example 2.1 and denote $X_{2}$ simply by $X$. Let $\nu: \mathbb{B}=\operatorname{Spec} \mathbb{C}[s] \rightarrow X / / D_{n}=$ Spec $\mathbb{C}[t, u] /\left(u^{2}-4 t^{n}\right)$ be the normalization, where $t=s^{2}$ and $u=2 s^{n}$, and let $F_{X}=\pi_{X}^{-1}(\nu(1))$. Then $F_{X} \cong D_{n} / H^{\prime}$, where $H^{\prime}=\mathbb{Z} / 2 \mathbb{Z}$ (the reflection subgroup) and $V_{n}$ is multiplicity free with respect to $H^{\prime}$. There is a $D_{n}$-equivariant morphism

$$
\begin{aligned}
\varphi^{X}: \mathbb{B} \times F_{X} & \rightarrow X, \\
(b, f) & \mapsto b f,
\end{aligned}
$$

which is an isomorphism from $(\mathbb{B}-\{0\}) \times F_{X}$ onto $X-\pi_{X}^{-1}(\nu(0))$. Note that the following diagram commutes:

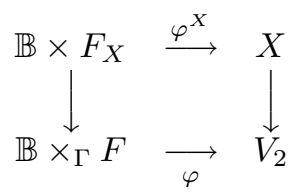

where the vertical maps are inclusions. Let $\mathfrak{m}_{X}=\operatorname{Mor}\left(F_{X} \text {, End } V_{n}\right)^{D_{n}}$. Then $\mathfrak{m}_{X}(\mathbb{B}):=\operatorname{Mor}\left(\mathbb{B}, \mathfrak{m}_{X}\right)$ is a free $\mathbb{C}[s]$-module with a grading induced from $\mathbb{C}[s]$. The $\mathbb{C}[X]^{D_{n}}$-module $\operatorname{Mor}\left(X \text {, End } V_{n}\right)^{D_{n}} \cong\left(\mathbb{C}[X] \otimes \text { End } V_{n}\right)^{D_{n}}$ inherits a grading from $\mathbb{C}[X] \subset \mathbb{C}\left[V_{2}\right]$. Note that $\mathfrak{m}_{X}(\mathbb{B})$ is considered as a $\mathbb{C}[X]^{D_{n}}$-module via $\nu$. The morphism $\varphi^{X}$ induces

$$
\varphi_{\#}^{X}: \operatorname{Mor}\left(X, \text { End } V_{n}\right)^{D_{n}} \rightarrow \operatorname{Mor}\left(\mathbb{B} \times F_{X}, \text { End } V_{n}\right)^{D_{n}}=\mathfrak{m}_{X}(\mathbb{B}),
$$

which is a $\mathbb{C}[X]^{D_{n}}$-homomorphism of degree 0 . Note that $\operatorname{Mor}\left(X, \text { End } V_{n}\right)^{D_{n}}$ is a finite free module over $\mathbb{C}[X]^{D_{n}}$. In fact, $\operatorname{Mor}\left(V_{2} \text {, End } V_{n}\right)^{D_{n}}$ is free over $\mathbb{C}[t, u]$ with a basis

$$
\begin{aligned}
\left\{\bar{A}_{0}=\right. & \left(\begin{array}{ll}
1 & 0 \\
0 & 1
\end{array}\right), \bar{A}_{1}=\left(\begin{array}{ll}
0 & 1 \\
1 & 0
\end{array}\right), \\
& \left.\left(\begin{array}{cc}
x^{n}-y^{n} & 0 \\
0 & -\left(x^{n}-y^{n}\right)
\end{array}\right), \quad\left(\begin{array}{cc}
0 & x^{n}-y^{n} \\
-\left(x^{n}-y^{n}\right) & 0
\end{array}\right)\right\} .
\end{aligned}
$$

Hence $\operatorname{Mor}\left(X, \text { End } V_{n}\right)^{D_{n}}$ is a free module over $\mathbb{C}[t, u] /\left(u^{2}-4 t^{n}\right)$ with a basis $\left\{\bar{A}_{0}, \bar{A}_{1}\right\}$. Let $\operatorname{Mor}\left(X, \text { End } V_{n}\right)_{1}^{D_{n}}$ (respectively $\mathfrak{m}_{X}(\mathbb{B})_{1}$ ) be the submodule of $\operatorname{Mor}\left(X \text {, End } V_{n}\right)^{D_{n}}$ (respectively $\left.\mathfrak{m}_{X}(\mathbb{B})\right)$ of elements with positive degrees. Then $\operatorname{Vec}_{D_{n}}\left(X, V_{n}\right)$ is isomorphic to the quotient module of $\mathfrak{m}_{X}(\mathbb{B})_{1}$ by

$$
\varphi_{\#}^{X} \operatorname{Mor}\left(X, \text { End } V_{n}\right)_{1}^{D_{n}} .
$$

The module $\mathfrak{m}_{X}(\mathbb{B})_{1}$ is free over $\mathbb{C}[s]$ with a basis

$$
\left\{\bar{C}_{0}=s\left(\begin{array}{ll}
1 & 0 \\
0 & 1
\end{array}\right), \bar{C}_{1}=s\left(\begin{array}{ll}
0 & 1 \\
1 & 0
\end{array}\right)\right\} .
$$

Since $\varphi_{\#}^{X}\left(t \bar{A}_{i}\right)=s \bar{C}_{i}$ and $\varphi_{\#}^{X}\left(u \bar{A}_{i}\right)=2 s^{n-1} \bar{C}_{i}$ for $i=0,1$,

$$
\operatorname{Vec}_{D_{n}}\left(X, V_{n}\right) \cong \mathfrak{m}_{X}(\mathbb{B})_{1} / \varphi_{\#}^{X} \operatorname{Mor}\left(X, \text { End } V_{n}\right)_{1}^{D_{n}} \cong \mathbb{C}^{n-1}
$$


with a basis $\left\{s^{2(j-1)} \bar{C}_{i} ; i=0,1,1 \leq j \leq(n-1) / 2\right\}$. The inclusions $\mathbb{B} \times F_{X} \hookrightarrow \mathbb{B} \times_{\Gamma} F$ and $X \hookrightarrow V_{2}$ give rise to a homomorphism

$$
\iota: \mathfrak{m}(\mathbb{B})_{1}^{\Gamma} / \varphi_{\#} \operatorname{Mor}\left(V_{2}, \text { End } V_{n}\right)_{1}^{O(2)} \rightarrow \mathfrak{m}_{X}(\mathbb{B})_{1} / \varphi_{\#}^{X} \operatorname{Mor}\left(X, \text { End } V_{n}\right)_{1}^{D_{n}},
$$

which corresponds to $i_{2}^{*} \circ d_{n}$. Since $\iota\left(t^{i-1} C_{1}\right)=s^{2(i-1)} \bar{C}_{1}$, it follows that $i_{2}^{*} \circ d_{n}$ is injective and $\operatorname{Im}\left(i_{2}^{*} \circ d_{n}\right) \cong \mathbb{C}^{(n-1) / 2}$ with a basis $\left\{s^{2(j-1)} \bar{C}_{1} ; 1 \leq j \leq(n-1) / 2\right\}$.

(2) From Proposition 3.1, there is a map

$$
\Phi_{2, n}: \operatorname{VAR}_{D_{n}}\left(V_{2}, V_{n}\right) \rightarrow \operatorname{Vec}_{D_{n}}\left(X_{2}, V_{n}\right) / \mathbb{C}^{*}
$$

From (1), $\operatorname{Im} i_{2}^{*}$ contains a subspace

$$
\bigoplus_{i=1}^{(n-1) / 2} W(2 i-1)
$$

In fact, $\operatorname{Im} i_{2}^{*} \cong \bigoplus_{i=1}^{(n-1) / 2} W(2 i-1)$ (cf. [18, III 3,4]). Hence the assertion follows.

(3) follows from (1) and Proposition 3.1.

Remark. From Theorem $3.2(1), d_{n}: \operatorname{Vec}_{O(2)}\left(V_{2}, V_{n}\right) \rightarrow \operatorname{Vec}_{D_{n}}\left(V_{2}, V_{n}\right)$ is an injection.

Let $\varepsilon$ be the 1-dimensional sign representation and let $\varepsilon^{m}$ be the direct sum of $m$ copies of $\varepsilon$. One can show by direct calculation that the composite map $\widetilde{i_{2}^{*}} \circ \widetilde{d_{n}}$ given by

$$
\begin{aligned}
\operatorname{Vec}_{O(2)}\left(V_{2}, V_{n} \oplus \mathbb{C}^{m_{1}} \oplus \varepsilon^{m_{2}}\right) & \stackrel{\widetilde{d_{n}}}{\rightarrow} \operatorname{Vec}_{D_{n}}\left(V_{2}, V_{n} \oplus \mathbb{C}^{m_{1}} \oplus \varepsilon^{m_{2}}\right) \\
& \stackrel{\widetilde{i_{2}^{*}}}{\longrightarrow} \operatorname{Vec}_{D_{n}}\left(X_{2}, V_{n} \oplus \mathbb{C}^{m_{1}} \oplus \varepsilon^{m_{2}}\right)
\end{aligned}
$$

is an injection. In fact, since the dimensions of $V_{2} / / O(2)$ and $X_{2} / / D_{n}$ are both equal to 1 , the map $\widetilde{i_{2}^{*}} \circ \widetilde{d_{n}}$ is a homomorphism of $\mathbb{C}$-vector groups. Since the generators of the $\mathbb{C}$-vector group $\operatorname{Vec}_{O(2)}\left(V_{2}, V_{n} \oplus \mathbb{C}^{m_{1}} \oplus \varepsilon^{m_{2}}\right)$, which is isomorphic to $\operatorname{Vec}_{O(2)}\left(V_{2}, V_{n}\right)$, do not vanish by the homomorphism $\tilde{i}_{2}^{*} \circ \widetilde{d_{n}}$ (cf. [7, VII 4], [18. III 5]), so $\widetilde{i_{2}^{*}} \circ \widetilde{d_{n}}$ is injective. The map

$$
\theta_{2}: \operatorname{Vec}_{D_{n}}\left(V_{2}, V_{n}\right) \rightarrow \operatorname{Vec}_{D_{n}}\left(V_{2}, V_{n} \oplus \mathbb{C}^{m_{1}} \oplus \varepsilon^{m_{2}}\right)
$$

sending $[E]$ to $\left[E \oplus \Theta_{\mathbb{C}^{m_{1}} \oplus \varepsilon^{m_{2}}}\right]$ induces a map

$$
\operatorname{VAR}_{D_{n}}\left(V_{2}, V_{n}\right) \rightarrow \operatorname{VAR}_{D_{n}}\left(V_{2}, V_{n} \oplus \mathbb{C}^{m_{1}} \oplus \varepsilon^{m_{2}}\right)
$$

which is the product map with $\mathbb{C}^{m_{1}} \times \varepsilon^{m_{2}}$.

Theorem 3.3. Let $n$ be odd and let $m_{1}$ and $m_{2}$ be non-negative integers. Then the map

$$
\begin{aligned}
\operatorname{VAR}_{O(2)}\left(V_{2}, V_{n}\right) & \rightarrow \operatorname{VAR}_{D_{n}}\left(V_{2}, V_{n}\right) \\
& \rightarrow \operatorname{VAR}_{D_{n}}\left(V_{2}, V_{n} \oplus \mathbb{C}^{m_{1}} \oplus \varepsilon^{m_{2}}\right)
\end{aligned}
$$

induced by $\theta_{2} \circ d_{n}$ is an injection.

Proof. Let $E, E^{\prime} \in \operatorname{Vec}_{O(2)}\left(V_{2}, V_{n}\right)$ be such that $E \times \mathbb{C}^{m_{1}} \times \varepsilon^{m_{2}} \cong E^{\prime} \times \mathbb{C}^{m_{1}} \times \varepsilon^{m_{2}}$ as $D_{n}$-varieties. Then applying Proposition 2.3 to $E \oplus \Theta_{\mathbb{C}^{m_{1}} \oplus \varepsilon^{m_{2}}}$ and $E^{\prime} \oplus \Theta_{\mathbb{C}^{m_{1}} \oplus \varepsilon^{m_{2}}}$ with $H=\mathbb{Z} / 2 \mathbb{Z}$ (the reflection subgroup) and $K=D_{n}$, we have

$$
\left.\left.\left(E \oplus \Theta_{\mathbb{C}^{m_{1}} \oplus \varepsilon^{m_{2}}}\right)\right|_{X_{2}} \cong\left(c^{*} E^{\prime} \oplus \Theta_{\mathbb{C}^{m_{1}} \oplus \varepsilon^{m_{2}}}\right)\right|_{X_{2}}
$$


as $D_{n}$-vector bundles, where $c$ is a scalar multiplication of $V_{2}$. Since $\widetilde{i_{2}^{*}} \circ \widetilde{d_{n}}$ is injective, $E \oplus \Theta_{\mathbb{C}^{m_{1}} \oplus \varepsilon^{m_{2}}} \cong c^{*} E^{\prime} \oplus \Theta_{\mathbb{C}^{m_{1}} \oplus \varepsilon^{m_{2}}}$ as $O(2)$-vector bundles. Since the Whitney sum with $\Theta_{\mathbb{C}^{m_{1}} \oplus \varepsilon^{m_{2}}}$ induces an isomorphism

$$
\operatorname{Vec}_{O(2)}\left(V_{2}, V_{n}\right) \cong \operatorname{Vec}_{O(2)}\left(V_{2}, V_{n} \oplus \mathbb{C}^{m_{1}} \oplus \varepsilon^{m_{2}}\right) \text {, }
$$

it follows that $E \cong c^{*} E^{\prime}$ as $O(2)$-vector bundles, and the assertion follows.

Remark. One of the first examples of non-linearizable actions by Schwarz is the $O(2)$-action on the total space of the non-trivial $E \in \operatorname{Vec}_{O(2)}\left(V_{2}, V_{3}\right)$. By Theorem 3.2 (3), the action of $D_{3}$ on $E$ is non-linearizable. Furthermore, by Theorem 3.3, the $D_{3}$-action on $E \times \mathbb{C}^{m_{1}} \times \varepsilon^{m_{2}}$ remains non-linearizable (cf. [3]). Since the map $\operatorname{Vec}_{O(2)}\left(V_{2}, V_{n}\right) \rightarrow \operatorname{Vec}_{O(2)}\left(V_{2}, V_{n} \oplus V_{1}\right)$ sending $[E]$ to $\left[E \oplus \Theta_{V_{1}}\right]$ is trivial [20], the $D_{3}$-action on $E \times V_{1}$ is linearizable.

By a method similar to the proof of Theorem 3.2, we can show the following.

Theorem 3.4 (cf. [16]). Let $m$ and $n$ be even and $m \leq n / 4$.

(1) The composite map $i_{1}^{*} \circ d_{n}: \operatorname{Vec}_{O(2)}\left(V_{1}, V_{m}\right) \rightarrow \operatorname{Vec}_{D_{n}}\left(X_{1}, V_{m}\right)$ is an isomorphism. Hence, $d_{n}: \operatorname{Vec}_{O(2)}\left(V_{1}, V_{m}\right) \rightarrow \operatorname{Vec}_{D_{n}}\left(V_{1}, V_{m}\right)$ is injective and $i_{1}^{*}: \operatorname{Vec}_{D_{n}}\left(V_{1}, V_{m}\right) \rightarrow \operatorname{Vec}_{D_{n}}\left(X_{1}, V_{m}\right)$ is surjective.

(2) The map

$$
\Phi_{1, m}: \operatorname{VAR}_{D_{n}}\left(V_{1}, V_{m}\right) \rightarrow \mathbb{P}_{*}(2 i ; 1 \leq i \leq m-1)
$$

is surjective.

(3) The map $\operatorname{VAR}_{O(2)}\left(V_{1}, V_{m}\right) \rightarrow \operatorname{VAR}_{D_{n}}\left(V_{1}, V_{m}\right)$ is injective. Hence, if $E \in$ $\operatorname{Vec}_{O(2)}\left(V_{1}, V_{m}\right)$ is a non-trivial $O(2)$-vector bundle, then the $D_{n}$-action on $E$ is non-linearizable.

Proof. (1) By 20], $\operatorname{Vec}_{O(2)}\left(V_{1}, V_{m}\right) \cong \mathbb{C}^{m-1}$ and by 8, $\operatorname{Vec}_{D_{n}}\left(X_{1}, V_{m}\right) \cong \mathbb{C}^{m-1}$. We can show that $i_{1}^{*} \circ d_{n}$ is an isomorphism directly as in the proof of Theorem 3.2 (1).

(2) By [8], $\operatorname{Vec}_{D_{n}}\left(X_{1}, V_{m}\right) \cong \bigoplus_{i=1}^{m-1} W(2 i)$. From this together with (1), the assertion follows.

(3) follows from (1) and Proposition 3.1.

Remarks. (1) When $m$ and $n$ are even and $n / 4<m<n / 2$, one can show that $\operatorname{Vec}_{D_{n}}\left(X_{1}, V_{m}\right) \cong \bigoplus_{i=1}^{n / 2-m-1} W(2 i)([8])$, and $i_{1}^{*} \circ d_{n}$ is a surjection. Hence $\Phi_{1, m}$ is a surjection from $\operatorname{VAR}_{D_{n}}\left(V_{1}, V_{m}\right)$ onto $\mathbb{P}_{*}(2 i ; 1 \leq i \leq n / 2-m-1)$.

(2) When $n$ is even, the Whitney sum maps

$$
\operatorname{Vec}_{O(2)}\left(V_{1}, V_{m}\right) \rightarrow \operatorname{Vec}_{O(2)}\left(V_{1}, V_{m} \oplus \mathbb{C}^{m_{1}} \oplus \varepsilon^{m_{2}}\right)
$$

and

$$
\operatorname{Vec}_{D_{n}}\left(X_{1}, V_{m}\right) \rightarrow \operatorname{Vec}_{D_{n}}\left(X_{1}, V_{m} \oplus \mathbb{C}^{m_{1}} \oplus \varepsilon^{m_{2}}\right)
$$

are trivial (cf. [20], [8]).

(3) Suppose $n$ is odd. Then the map

$$
i_{1}^{*} \circ d_{n}: \operatorname{Vec}_{O(2)}\left(V_{1}, V_{m}\right) \rightarrow \operatorname{Vec}_{D_{n}}\left(X_{1}, V_{m}\right)
$$

is injective and

$$
\operatorname{Im}\left(i_{1}^{*} \circ d_{n}\right) \cong \bigoplus_{i=1}^{m-1} W(2 i)
$$


(cf. [18]). Hence, when $(m, n)>1$, $\operatorname{VAR}_{O(2)}\left(V_{1}, V_{m}\right) \rightarrow \operatorname{VAR}_{D_{n}}\left(V_{1}, V_{m}\right)$ is injective.

Consider the commutative diagram for $n$ odd:

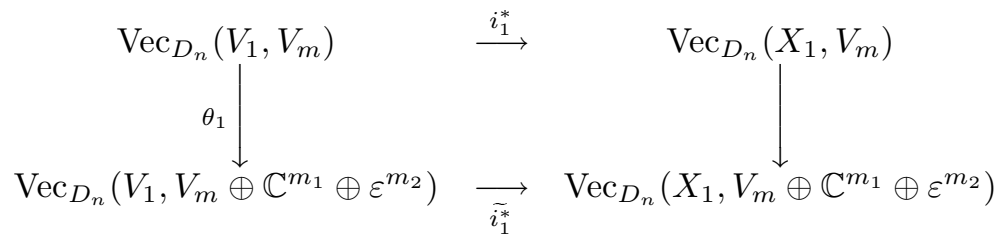

where the vertical maps are the Whitney sum maps with $\Theta_{\mathbb{C}^{m_{1}} \oplus \varepsilon^{m_{2}}}$. By [18],

$$
\operatorname{Im} i_{1}^{*} \cong\left(\bigoplus_{i=1}^{2 m-1} W(i)\right) \oplus\left(\bigoplus_{i=1}^{(n-1) / 2-2 m} W(2 m-1+2 i)\right)
$$

for $m<n / 4$,

$$
\operatorname{Im} i_{1}^{*} \cong\left(\bigoplus_{i=1}^{n-2 m-1} W(i)\right) \oplus\left(\bigoplus_{i=1}^{2 m-(n+1) / 2} W(n-2 m-1+2 i)\right)
$$

for $n / 4<m<n / 2$, and

$$
\operatorname{Im}\left(\widetilde{i_{1}^{*}} \circ \theta_{1}\right) \cong \bigoplus_{i=1}^{(n-2 m-1) / 2} W(2 i-1) .
$$

Hence we obtain the following by applying Proposition 2.3.

Theorem 3.5. Suppose that $n$ is odd and $(m, n)>1$. Then the image of $\Phi_{1, m}$ is isomorphic to the weighted projective space $\mathbb{P}_{*}((n-5) / 2)$ with a vertex. The space $\mathbb{P}_{*}((n-5) / 2)$ is of dimension $(n-5) / 2$ and contains the weighted projective space $\mathbb{P}(2 i-1 ; 1 \leq i \leq(n-2 m-1) / 2)$ whose inverse image under $\Phi_{1, m}$ consists of elements $E$ such that the $D_{n}$-action on $E \times \mathbb{C}^{m_{1}} \times \varepsilon^{m_{2}}$ is non-linearizable.

Remark. Mederer [18] showed that $\operatorname{Vec}_{D_{3}}\left(V_{1}, V_{1}\right) \cong \Omega_{\mathbb{C}}$, the module of Kähler differentials of $\mathbb{C}$ over $\mathbb{Q}$, and furthermore, there is a surjection from Ker $i_{1}^{*}$ in the above diagram for $n \geq 5$ to $\operatorname{Vec}_{D_{3}}\left(V_{1}, V_{1}\right)$. Hence $\operatorname{Vec}_{D_{n}}\left(V_{1}, V_{m}\right)(n$ odd; $n \geq 5)$ contains a space of uncountably-infinite dimension. Proposition 2.3 is, to our regret, not useful for classifying the $D_{n}$-actions derived from $\operatorname{Ker} i_{k}^{*}$ or $\operatorname{Vec}_{D_{3}}\left(V_{1}, V_{1}\right)$.

Suppose $n$ is odd, and classify the $D_{n}$-actions derived from $\operatorname{Vec}_{D_{n}}\left(V_{2} \oplus \varepsilon^{m}, V_{n}\right)$. By applying Proposition 2.3 for $H=\mathbb{Z} / 2 \mathbb{Z}$ and $K=D_{n}$, we obtain a surjection from $\operatorname{VAR}_{D_{n}}\left(V_{2} \oplus \varepsilon^{m}, V_{n}\right)$ to the orbit space of $\operatorname{Im} i_{2, m}^{*}$ under the action of $\mathbb{C}^{*}$, where $i_{2, m}^{*}: \operatorname{Vec}_{D_{n}}\left(V_{2} \oplus \varepsilon^{m}, V_{n}\right) \rightarrow \operatorname{Vec}_{D_{n}}\left(X_{2}, V_{n}\right)$ is the restriction induced by $i_{2, m}: X_{2} \hookrightarrow V_{2} \oplus \varepsilon^{m}$. Let $i_{m}: V_{2} \rightarrow V_{2} \oplus \varepsilon^{m}$ be the inclusion. Then $i_{2, m}^{*}=i_{2}^{*} \circ i_{m}^{*}$. Since $i_{m}^{*}$ is a surjection, $\operatorname{Im} i_{2, m}^{*}=\operatorname{Im} i_{2}^{*}$. Since $\operatorname{Im} i_{2}^{*} \cong \bigoplus_{i=1}^{(n-1) / 2} W(2 i-1)$ (cf. the proof of Theorem $3.2(2))$, we have a surjection

$$
\operatorname{VAR}_{D_{n}}\left(V_{2} \oplus \varepsilon^{m}, V_{n}\right) \rightarrow \mathbb{P}_{*}(2 i-1 ; 1 \leq i \leq(n-1) / 2) .
$$

Theorem 3.6. Let $m$ be a non-negative integer and let $n$ be odd. Then there is a surjection from $\operatorname{VAR}_{D_{n}}\left(V_{2} \oplus \varepsilon^{m}, V_{n}\right)$ onto $\mathbb{P}_{*}(2 i-1 ; 1 \leq i \leq(n-1) / 2)$.

Remark. Let $l$ be a non-negative integer and let $(m, n)>1$. Then one obtains a similar result for $\operatorname{VAR}_{D_{n}}\left(V_{1} \oplus \varepsilon^{l}, V_{m}\right)$. 


\section{REFERENCES}

1. H. Bass, W. Haboush, Some equivariant K-theory of affine algebraic group actions, Comm. Algebra 15 (1987), 181-217. MR 88g:14013

2. A. Bialynicki-Birula, Remarks on the action of an algebraic torus on $k^{n}$, I and II, Bull. Acad. Pol. Sci. 14 (1966), 177-181, and 15 (1967), 123-125.

3. G. Freudenburg, L. Moser-Jauslin, A nonlinearizable action of $S_{3}$ on $\mathbb{C}^{4}$, Ann. Inst. Fourier, Grenoble 52 (2002), 133-143. MR 2002j:14068

4. S. Kaliman, M. Koras, L. Makar-Limanov, P. Russell, $\mathbb{C}^{*}$-actions on $\mathbb{C}^{3}$ are linearizable, Electronic Research Announcements of the AMS 3 (1997), 63-71. MR 98i:14046

5. F. Knop, Nichtlinearisierbare Operationen halbeinfacher Gruppen auf affinen Räumen, Invent. Math. 105 (1991), 217-220. MR 92c:14046

6. H. Kraft, $G$-vector bundles and the Linearization Problem, in Group Actions and Invariant Theory, CMS Conference Proceedings, vol. 10, Amer. Math. Soc., Providence, RI, 1989, pp. 111-123. MR 90j:14062

7. H. Kraft, G.W. Schwarz, Reductive group actions with one-dimensional quotient, Publ. Math. IHES 76 (1992), 1-97. MR 94e:14065

8. K. Masuda, Moduli of equivariant algebraic vector bundles over affine cones with one dimensional quotient, Osaka J. Math. 32 (1995), 1065-1085. MR 97k:14049

9. K. Masuda, Moduli of equivariant algebraic vector bundles over a product of affine varieties, Duke Math. J. 88 (1997), 181-199. MR 99b:14011

10. K. Masuda, Moduli of algebraic $S L_{3}$-vector bundles over adjoint representaion, Osaka J. Math. 38 (2001), 501-506. MR 2002g:14069

11. M. Masuda, L. Moser-Jauslin, T. Petrie, Equivariant algebraic vector bundles over representations of reductive groups: Applications, Proc. Natl. Acad. Sci. 88 (1991), 9065-9066. MR 92j:14059b

12. M. Masuda, L. Moser-Jauslin, T. Petrie, The equivariant Serre problem for abelian groups, Topology 35 (1996), 329-334. MR 97a:20074

13. M. Masuda, L. Moser-Jauslin, T. Petrie, Equivariant algebraic vector bundles over cones with smooth one dimensional quotient, J. Math. Soc. Japan 50 (1998), 379-414. MR 99k:14079

14. M. Masuda, T. Nagase, Equivariant algebraic vector bundles over adjoint representations, Osaka J. Math. 32 (1995), 701-708. MR 96k:14014

15. M. Masuda, T. Petrie, Equivariant algebraic vector bundles over representations of reductive groups: Theory, Proc. Nat. Acad. Sci. 88 (1991), 9061-9064. MR 92j:14059a

16. M. Masuda, T. Petrie, Stably trivial equivariant algebraic vector bundles, J. Amer. Math. Soc. 8 (1995), 687-714. MR 95j:14067

17. M. Masuda, T. Petrie, Algebraic families of $O(2)$-actions on affine space $\mathbb{C}^{4}$, Proc. of Symp. in Pure Math., vol. 56, Amer. Math. Soc., Providence, RI, 1994, pp. 347-354. MR 95f:14092

18. K. Mederer, Moduli of $G$-equivariant vector bundles, Ph.D. thesis, Brandeis University (1995).

19. D. Quillen, Projective modules over polynomial rings, Invent. Math., 36 (1976), 167-171. MR 55:337

20. G.W. Schwarz, Exotic algebraic group actions, C. R. Acad. Sci. Paris 309 (1989), 89-94. MR 91b:14066

21. A. Suslin, Projective modules over a polynomial ring, Soviet Math. Doklady 17 (1976), 11601164 .

Mathematical Science II, Himeji Institute of Technology, 2167 Shosha, Himeji 6712201, JAPAN

E-mail address: kayo@sci.himeji-tech.ac.jp 